\title{
Structural distortions of frustrated quantum spin lattices in high magnetic fields
}

\author{
Oleg Derzhko ${ }^{1,2}$, Johannes Richter ${ }^{2}$ and Jörg Schulenburg ${ }^{3}$ \\ ${ }^{1}$ Institute for Condensed Matter Physics, National Academy of Sciences of Ukraine, \\ 1 Svientsitskii Street, L'viv-11, 79011, Ukraine \\ ${ }^{2}$ Institut für Theoretische Physik, Universität Magdeburg, \\ P.O. Box 4120, D-39016 Magdeburg, Germany \\ ${ }^{3}$ Universitätsrechenzentrum, Universität Magdeburg, \\ P.O. Box 4120, D-39016 Magdeburg, Germany
}

September 28, 2018

\begin{abstract}
We study the stability of some strongly frustrated antiferromagnetic spin lattices in high magnetic fields against lattice distortions. In particular, we consider a spin- $s$ anisotropic Heisenberg antiferromagnet on the square-kagomé and kagomé lattices. The independent localized magnons embedded in a ferromagnetic environment, which are the ground state at the saturation field, imply lattice instabilities for appropriate lattice distortions fitting to the structure of the localized magnons. We discuss in detail the scenario of this spin-Peierls instability in high magnetic fields which essentially depends on the values of the exchange interaction anisotropy $\Delta$ and spin $s$.
\end{abstract}

PACS number(s): $75.10 . J m, 75.45 .+\mathrm{j}$

Keywords: frustrated antiferromagnets, high magnetic fields, spin-Peierls instability

\section{Introduction. Localized magnons}

The square-kagomé and kagomé Heisenberg antiferromagnets have attracted much interest in recent times because of interplay between quantum fluctuations and frustrated lattice structure [1, 2, 3, 4, 5. Recently, for a wide class of frustrated spin lattices, which includes both the squarekagomé and kagomé lattices, exact eigenstates consisting of independent localized magnons in a ferromagnetic environment have been found $[6,7,8$. They become ground states if the saturating magnetic field is applied and lead to a macroscopic jump in the zero-temperature magnetization 

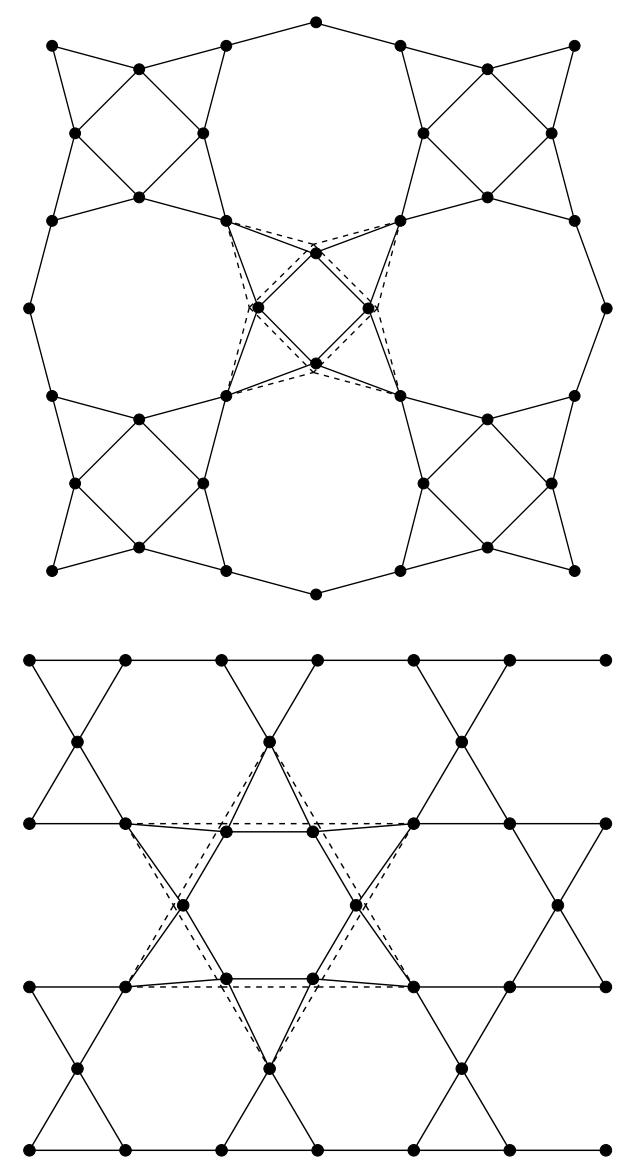

Figure 1: Square-kagomé lattice with one distorted square (top) and kagomé lattice with one distorted hexagon (bottom) which can host localized magnons. The parts of the lattices before distortions are shown by dashed lines. All bonds in the lattice before distortions have the same length.

curve just below saturation. Moreover, very recently we have examined a field-tuned instability of the square-kagomé and kagomé spin lattices with respect to lattice distortions through a magnetoelastic mechanism [9] reporting rigorous analytical results completed by large-scale exact diagonalization data for lattices up to $N=54$ sites [10]. That study was restricted mainly to the spin- $\frac{1}{2}$ isotropic Heisenberg antiferromagnet. Since often one meets kagomé materials with $s>\frac{1}{2}$ (for instance, spin- $\frac{3}{2}$ kagomé-like compound $\mathrm{Ba}_{2} \mathrm{Sn}_{2} \mathrm{ZnCr}_{7 p} \mathrm{Ga}_{10-7 p} \mathrm{O}_{22}$ with a comparably small exchange constant of about $37 \ldots 40 \mathrm{~K}$ [1]) it is useful to go beyond the case $s=\frac{1}{2}$ and $\Delta=1$. In this paper we extend our previous results for the spin quantum numbers $s>\frac{1}{2}$ and exchange interaction anisotropy $\Delta \neq 1$ demonstrating that a scenario of the discussed earlier magnetic-field induced lattice instability may essentially depend on the values of $s$ and $\Delta$.

To be specific, we consider the square-kagomé lattice (Fig. 1, top) and the kagomé lattice 
(Fig. 1] bottom). The Hamiltonian of $N$ quantum spins reads

$$
H=\sum_{(n m)} J_{n m}\left(\frac{1}{2}\left(s_{n}^{+} s_{m}^{-}+s_{n}^{-} s_{m}^{+}\right)+\Delta s_{n}^{z} s_{m}^{z}\right)-h S^{z} .
$$

Here the sum runs over the bonds (edges) which connect the sites (vertices) occupied by spins for the lattice under consideration, $J_{n m}>0$ are the antiferromagnetic exchange constants between the sites $n$ and $m, \Delta \geq 0$ is the anisotropy parameter, $h$ is the external magnetic field, and $S^{z}=\sum_{n} s_{n}^{z}$ is the $z$-component of the total spin. We assume that all bonds in the lattice without distortion have the same length and hence all exchange constants have the same value $J$.

From Refs. [6, 7, 8, 5] we know that independent localized one-magnon states embedded in a ferromagnetic background are exact ground states of the Hamiltonian (11) at saturation field for the considered models.

More specifically, by direct computation one can check that

$$
\begin{gathered}
|1\rangle=\frac{1}{\sqrt{4}}(|s-1, s, s, s\rangle-|s, s-1, s, s\rangle \\
+|s, s, s-1, s\rangle-|s, s, s, s-1\rangle)|\ldots s \ldots\rangle
\end{gathered}
$$

and

$$
\begin{gathered}
|1\rangle=\frac{1}{\sqrt{6}}(|s-1, s, s, s, s, s\rangle-|s, s-1, s, s, s, s\rangle+|s, s, s-1, s, s, s\rangle \\
-|s, s, s, s-1, s, s\rangle+|s, s, s, s, s-1, s\rangle-|s, s, s, s, s, s-1\rangle)|\ldots s \ldots\rangle
\end{gathered}
$$

are one-magnon eigenstates of the Hamiltonian (11) on the square-kagomé and kagomé lattices. Here $s$ or $s-1$ denote the value of $s_{n}^{z}$, the omitted site indices in the first multiplier in the r.h.s. of Eq. (2) (Eq. (3)) run along a square (hexagon) trapping cell, and the second multiplier in the r.h.s. of Eqs. (2) and (3), $|\ldots s \ldots\rangle$, stands for the embedding fully polarized ferromagnetic environment. The corresponding energies $(h=0)$ of the one-magnon states (2) and (3) are

$$
E_{1}=-2 s J+2 s(2 s-1) \Delta J+2 s(4 s-1) \Delta J+(2 N-12) s^{2} \Delta J
$$

and

$$
E_{1}=-2 s J+2 s(3 s-1) \Delta J+2 s(6 s-1) \Delta J+(2 N-18) s^{2} \Delta J
$$

We separate in Eqs. (41), (15) the contributions to the energy from those bonds which form a magnon trapping cell (first and second terms), from the bonds connecting this cell with the environment (third terms) and from the ferromagnetic environment (fourth terms). The considered lattices may contain $n=1, \ldots, n_{\max }$ localized magnons where $n_{\max }=\frac{1}{6} N$ or $\frac{1}{9} N$ 
for the square-kagomé and kagomé lattices, respectively. Each magnon decreases the total $S^{z}$ by one and a localized magnon state with $n$ independent magnons has $S^{z}=s N-n$. In the presence of an external field $h \neq 0$ the energy $E\left(S^{z}, h\right)$ can be obtained from the energy without field $E\left(S^{z}\right)$ through the relation $E\left(S^{z}, h\right)=E\left(S^{z}\right)-h S^{z}$.

Under quite general assumptions it was proved [6, 12] that the localized magnon states have lowest energies in the corresponding sectors of total $S^{z}$. As a result, these states become ground states at the saturation field. More specifically, the ground-state energy in the presence of a field is given by $E_{0}(h)=E_{\min }\left(S^{z}\right)-h S^{z}$ and the ground-state magnetization $S^{z}$ is determined from the equation $h=E_{\min }\left(S^{z}\right)-E_{\min }\left(S^{z}-1\right)$. Since for $S^{z}=s N, \ldots, s N-n_{\max }$ the localized magnon states are the lowest states, one has

$$
E_{\min }\left(S^{z}\right)=2 N s^{2} \Delta J-n \epsilon_{1}=2 N s^{2} \Delta J-N s \epsilon_{1}+\epsilon_{1} S^{z}
$$

where

$$
\epsilon_{1}=2 s(1-(2 s-1) \Delta) J-2 s(4 s-1) \Delta J+12 s^{2} \Delta J=2 s(1+2 \Delta) J
$$

and

$$
\epsilon_{1}=2 s(1-(3 s-1) \Delta) J-2 s(6 s-1) \Delta J+18 s^{2} \Delta J=2 s(1+2 \Delta) J
$$

for the square-kagomé and kagomé lattices, respectively. Due to the linear relation between $E_{\min }$ and $S^{z}$ (6) one has a complete degeneracy of all localized magnon states at the saturation field $h=h_{1}=\epsilon_{1}$, i.e. the energy is $2 N s^{2} \Delta J-N s \epsilon_{1}$ at $h=h_{1}$ for all $s N-n_{\max } \leq S^{z} \leq s N$. Consequently, the zero-temperature magnetization $S^{z}$ jumps between the saturation value $s N$ and the value $s N-\frac{1}{6} N\left(s N-\frac{1}{9} N\right)$ for the square-kagomé (kagomé) lattice.

Finally, we mention that the localization of magnon in a finite area has some relation to the flat one-magnon dispersion. Some rigorous results for the flat-band electronic systems have been reported in Refs. 13, 14, 15.

\section{Spin-Peierls instability}

To check the lattice stability of the considered systems with respect to a spin-Peierls mechanism we assume a small lattice deformation which preserves the symmetry of the cell which hosts the localized magnon (in this case the independent localized magnon states remain the exact eigenstates) and analyze the change in the total energy. The corresponding deformations are shown in Fig. 1. For the square-kagomé lattice (Fig. 11. top) the deformations lead to the following changes in the exchange interactions: $J \rightarrow(1+\sqrt{2} \delta) J$ (along the edges of the square) 
and $J \rightarrow\left(1-\frac{1}{4} \sqrt{2}(\sqrt{3}-1) \delta\right) J$ (along the two edges of the triangles attached to the square), where the quantity $\delta$ is proportional to the displacement of the atoms and the change in the exchange integrals due lattice distortions is taken into account in first order in $\delta$. For the kagomé lattice (Fig. 1 bottom) one has $J \rightarrow(1+\delta) J$ (along the edges of the hexagon) and $J \rightarrow\left(1-\frac{1}{2} \delta\right) J$ (along the two edges of the triangles attached to the hexagon). Note, that the lattice distortions shown in Fig. 1 correspond to $\delta>0$. The magnetic energies (4) and (5) are changed by distortions by $\left(-2 \sqrt{2} s+2 \sqrt{2} s(2 s-1) \Delta-\frac{\sqrt{3}-1}{\sqrt{2}} s(4 s-1) \Delta\right) \delta J$ and $-s(2+\Delta) \delta J$, respectively. Note that the linear with respect to $\delta$ change of the magnetic ground-state energies has a simple reason: the magnetic ground-state energy for $s N-n_{\max } \leq S^{z}<s N$ contains a sum of contributions coming from local lattice regions each of which varies linearly with $\delta$. The elastic energy increases in harmonic approximation by $2(6-\sqrt{3}) \alpha \delta^{2}$ (square-kagomé) and by $9 \alpha \delta^{2}$ (kagomé). The parameter $\alpha$ is proportional to the elastic constant of the lattice. The changes of total energies due to distortions read

$$
\Delta \mathrm{E}_{1}=\left(-2 \sqrt{2} s+2 \sqrt{2} s(2 s-1) \Delta-\frac{\sqrt{3}-1}{\sqrt{2}} s(4 s-1) \Delta\right) \delta J+2(6-\sqrt{3}) \alpha \delta^{2}
$$

and

$$
\Delta \mathrm{E}_{1}=-s(2+\Delta) \delta J+9 \alpha \delta^{2}
$$

where for $n$ independent localized magnons trapped by distorted cells these results have to be multiplied by $n$. Here comes the first important conclusion. For the kagomé lattice $\Delta \mathrm{E}_{1}$ (10) decreases at small $\delta>0$ for any $\Delta \geq 0$ and any $s$, thus implying a spin-Peierls instability with the distorted trapping cells like in Fig. 1, bottom. In contrast, for the square-kagomé lattice with $s>\frac{1}{2}$ the sign of $\delta$ which provides a decrease of $\Delta \mathrm{E}_{1}$ (9) for the small lattice distortions depends on the anisotropy parameter $\Delta: \delta>0$ if $\Delta<\Delta^{\star}(s)$ (with $\Delta^{\star}(1) \approx 2.21748, \Delta^{\star}\left(\frac{3}{2}\right) \approx 0.92171$ etc; $\Delta^{\star}(s) \sim \frac{1}{s}$ as $\left.s \rightarrow \infty\right)$ but $\delta<0$ if $\Delta>\Delta^{\star}(s)$. This can be understood as a result of interplay between the contributions of transverse and $z z$ correlations along different bonds for the square-kagomé lattice geometry.

Let us discuss the scenario of spin-Peierls instability for the square-kagomé lattice with $\Delta<\Delta^{\star}(s)$ and for the kagomé lattice. Consider a magnetic field above the saturation field $h_{1}$. For the corresponding fully polarized ferromagnetic state a lattice distortion is not favorable. Decreasing $h$ till $h_{1}$ the homogeneous ferromagnetic state transforms into the "distorted magnon crystal". Further, numerical analysis suggests that both spin-1 systems exhibit a magnetization plateau between $h_{1}$ and $h_{2}<h_{1}$ at $S^{z}=s N-n_{\max }$. Calculating the plateau width $\Delta h=h_{1}-h_{2}$ for spin-1 isotropic (i.e. $\Delta=1$ ) finite systems of $N=24,30$ (square-kagomé) and $N=27,36,45$ 
(kagomé) for the undistorted lattice $\left(h_{2}\right.$ is obtained by $h_{2}=E_{\min }\left(S^{z}=s N-n_{\max }\right)-E_{\min }\left(S^{z}=\right.$ $\left.\left.s N-n_{\max }-1\right)\right)$ and using a $\frac{1}{N}$ finite-size extrapolation we find some indications for a finite $\Delta h \approx 0.5 J(\Delta h \approx 0.1 J)$ for the square-kagomé (kagomé) lattice in the thermodynamic limit. We also mention here that on the basis of general arguments [16, 17] one may expect the magnon crystal state to have gapped excitations that is related to the magnetization plateau at $S^{z}=s N-n_{\max } \cdot$

Now the question arises whether the lattice distortion under consideration is stable below this plateau, i.e., for $S^{z}<s N-n_{\max }$. We discuss the question again for spin-1 finite systems of size $N=24$ (square-kagomé) and $N=36$ (kagomé) with $n_{\max }$ distorted squares/hexagons. We calculate the magnetic energy for zero and small distortion parameter $\delta$ for different values of $S^{z}$. Adopting for the magnetic energy the ansatz

$$
E_{\min }\left(S^{z}, \delta\right)=E_{\min }\left(S^{z}, 0\right)+A \delta^{p}
$$

and taking $\delta$ of the order of $10^{-4}$ we estimate the exponent $p$ from the numerical results. Evidently, the lattice may become unstable if the magnetic energy (111) decreases with the exponent $p<2$ whereas $p \geq 2$ indicates lattice stability. Interestingly, in the sector of $S^{z}$ just below the magnon crystal, $S^{z}=s N-n_{\max }-1$ (i.e. as $h$ becomes smaller than $h_{2}$ ), the spin-1 square-kagomé and kagomé lattices show different behavior. For the kagomé lattices with $N=36$ we do not find a lattice instability. Note that this is in agreement with the situation for $s=\frac{1}{2}$ [10]. Contrary to that, for the square-kagomé lattice with $N=24$ we find a lattice instability for $\delta>0$ also in the sector $S^{z}=s N-n_{\max }-1=19$ in case of small enough anisotropy $\Delta<\tilde{\Delta}(1) \approx 1.6$. Again if $\Delta>\tilde{\Delta}(1)$ the favorable lattice distortion is characterized by $\delta<0$. Since we know from Ref. [10 that for the spin $s=\frac{1}{2}$ square-kagomé lattice the spin-Peierls instability can be observed even for lower $S^{z}<s N-n_{\max }-1$, we check this also for the spin $s=1$ square-kagomé lattice with $N=24$. As for $S^{z}=19$ we find a lattice instability for $\delta>0$ only for small enough anisotropy $\Delta \lesssim 1.0$ for $S^{z}=18$ and $\Delta \lesssim 0.51$ for $S^{z}=17$, i.e. anisotropy parameter $\Delta$ below which this instability occurs becomes smaller when $S^{z}$ (i.e. the magnetic field $h$ ) is diminished. Thus, we arrive at the second important conclusion. For the spin $s=1$ kagomé lattice with any $\Delta \geq 0$ the spin-Peierls instability is favorable only for $s N-n_{\max } \leq S^{z}<s N$ and the distortion disappears for $h<h_{2}$. In contrast, the distorted spin-1 square-kagomé lattice with the sufficiently small anisotropy parameter $0 \leq \Delta<\tilde{\Delta}(1)$ remains stable for smaller $h<h_{2}$. The spin-1 square-kagomé lattice with $\tilde{\Delta}(1) \leq \Delta<\Delta^{\star}(1)$ exhibits more intricate behavior: the parameter $\delta$ characterizing the lattice distortion at the saturation changes its sign for $h<h_{2}$. 
As in the spin- $\frac{1}{2}$ case [10] the saturation field in the distorted lattice is shifted to higher values that provides a hysteresis phenomenon in the vicinity of saturation field.

\section{Concluding remarks}

There is an increasing number of synthesized quantum kagomé magnets often with large values of spin (unfortunately, however, the available at present materials are not perfect kagomé Heisenberg antiferromagnets) and we may expect that further such materials will be synthesized. With our analysis we are pointing out that the efforts in this direction are worthwhile also because of a new effect: the spin-Peierls instability in a strong magnetic field. From the point of view of possible experiments, it is important to bear in mind the following remarks. First, large values

of $s$ leads to decrease of a relative plateau width $\frac{\Delta h}{h_{1}}$. For instance, we have $\frac{\Delta h}{h_{1}} \approx 0.11$ for $s=\frac{1}{2}$ [10] and $\frac{\Delta h}{h_{1}} \approx 0.08$ for $s=1$ (undistorted square-kagomé, $\Delta=1$ ); $\frac{\Delta h}{h_{1}} \approx 0.023$ for $s=\frac{1}{2}$ [10] and $\frac{\Delta h}{h_{1}} \approx 0.017$ for $s=1$ (undistorted kagomé, $\Delta=1$ ). Thus, we need materials with not too large $s$ for which the plateau width is not extremely small. Second, we need materials with comparably small exchange constant $J$ to have experimentally accessible saturation field $h_{1}$. Third, the presented consideration refers to ideal kagomé geometry. We have not discussed the effects of deviation from the perfect kagomé geometry on the predicted spin-Peierls instability in high magnetic fields. Nevertheless we may expect that the effects of localized magnon states will survive (see, for example, Ref. [18]). Fourth, the reported analysis is performed within the frames of the adiabatic treatment of the spin-Peierls instability. The problem becomes much more difficult when the nonadiabatic effects are taken into account.

To summarize, we have examined a spin-Peierls instability in strong magnetic fields for two frustrated spin-s anisotropic Heisenberg antiferromagnets hosting independent localized magnons demonstrating cumulative effects of exchange interaction anisotropy, spin value and lattice geometry.

The present study was supported by the DFG (Project No. 436 UKR 17/17/03). O. D. and J. R. acknowledge the kind hospitality of the MPIPKS, Dresden in the spring of 2004. O. D. is grateful to the ICTP, Trieste for the kind hospitality during the Workshop on Novel States and Phase Transitions in Highly Correlated Matter (12 July - 23 July, 2004). The paper was presented at the Joint European Magnetic Symposia JEMS‘04 (September 5-10, 2004, Dresden, Germany). O. D. thanks the Organizers of the JEMS`04 for the grant for participation in the conference. 


\section{References}

[1] R. Siddharthan and A. Georges, Phys. Rev. B 65, 014417 (2001); P. Tomczak and J. Richter, J. Phys. A 36, 5399 (2003); J. Richter, J. Schulenburg, P. Tomczak, D. Schmalfuß, arXiv:cond-mat/0411673.

[2] P. Lecheminant, B. Bernu, C. Lhuillier, L. Pierre, and P. Sindzingre, Phys. Rev. B 56, 2521 (1997); Ch. Waldtmann, H.-U. Everts, B. Bernu, C. Lhuillier, P. Sindzingre, P. Lecheminant, and L. Pierre, Eur. Phys. J. B 2, 501 (1998); F. Mila, Phys. Rev. Lett. 81, 2356 (1998); M. E. Zhitomirsky and H. Tsunetsugu, Phys. Rev. B 70, 100403(R) (2004).

[3] C. Lhuillier and G. Misguich, in "High Magnetic Fields: Applications in Condensed Matter Physics and Spectroscopy", C. Berthier, L. P. Lévy, G. Martinez, Eds. (Lecture Notes in Physics, 595) (Springer, Berlin, 2002), pp.161-190; arXiv:cond-mat/0109146

[4] G. Misguich and C. Lhuillier, in "Frustrated Spin Systems", H. T. Diep, Ed. (World Scientific, Singapore, 2005), pp.229-306; arXiv:cond-mat/0310405

[5] J. Richter, J. Schulenburg, and A. Honecker, in "Quantum Magnetism", U. Schollwöck, J. Richter, D. J. J. Farnell, R. F. Bishop, Eds. (Lecture Notes in Physics, 645) (Springer, Berlin, 2004), pp.85-153.

[6] J. Schnack, H.-J. Schmidt, J. Richter, and J. Schulenburg, Eur. Phys. J. B 24, 475 (2001).

[7] J. Schulenburg, A. Honecker, J. Schnack, J. Richter, and H.-J. Schmidt, Phys. Rev. Lett. 88, 167207 (2002).

[8] J. Richter, J. Schulenburg, A. Honecker, J. Schnack, and H.-J. Schmidt, J. Phys.: Condens. Matter 16, S779 (2004).

[9] The effect of a magnetoelastic coupling in frustrated antiferromagnets at zero field is discussed in several papers, e.g., F. Becca, F. Mila, and D. Poilblanc, Phys. Rev. Lett. 91, 067202 (2003); F. Becca and F. Mila, Phys. Rev. Lett. 89, 037204 (2002); P. Carretta, N. Papinutto, C. B. Azzoni, M. C. Mozzati, E. Pavarini, S. Gonthier, and P. Millet, Phys. Rev. B 66, 094420 (2002); S.-H. Lee, C. Broholm, T. H. Kim, W. Ratcliff II, and SW. Cheong, Phys. Rev. Lett. 84, 3718 (2000); Y. Yamashita and K. Ueda, Phys. Rev. Lett. 85, 4960 (2000); A. Keren and J. S. Gardner, Phys. Rev. Lett. 87, 177201 (2001); O. Tchernyshyov, R. Moessner, and S. L. Sondhi, Phys. Rev. Lett. 88, 067203 (2002); O. Tchernyshyov, R. Moessner, and S. L. Sondhi, Phys. Rev. B 66, 064403 (2002). 
[10] J. Richter, O. Derzhko, and J. Schulenburg, Phys. Rev. Lett. 93, 107206 (2004).

[11] D. Bono, P. Mendels, G. Collin, and N. Blanchard, Phys. Rev. Lett. 92, 217202 (2004).

[12] H.-J. Schmidt, J. Phys. A 35, 6545 (2002).

[13] A. Mielke, J. Phys. A 24, 3311 (1991); A. Mielke, J. Phys. A 25, 4335 (1992).

[14] H. Tasaki, Phys. Rev. Lett. 69, 1608 (1992).

[15] H. Tasaki, J. Phys.: Condens. Matter 10, 4353 (1998); arXiv:cond-mat/9512169;

H. Tasaki, Prog. Theor. Phys. 99, 489 (1998); arXiv:cond-mat/9712219.

[16] T. Momoi and K. Totsuka, Phys. Rev. B 61, 3231 (2000).

[17] M. Oshikawa, Phys. Rev. Lett. 84, 1535 (2000).

[18] O. Derzhko and J. Richter, Phys. Rev. B 70, 104415 (2004). 